\title{
USING ACTIVE LEARNING IN HYBRID LEARNING ENVIRONMENTS
}

\author{
Demazière C \\ Chalmers University of Technology \\ Department of Physics, Division of Subatomic and Plasma Physics \\ SE-412 96 Gothenburg, Sweden \\ demaz@chalmers.se
}

\begin{abstract}
In this paper, an innovative pedagogical approach relying on flipped classroom and offered in a hybrid learning environment combining on-site and off-site attendees is proposed. The setup is furthermore tested on two short courses offered at Chalmers University of Technology and analyzed using student course evaluation questionnaires. Several elements constitute the backbone of the courses. Such elements are either offered in an asynchronous fashion or in a synchronous fashion. The asynchronous elements are made of textbooks specifically written for the respective courses, pre-recorded short webcasts explaining the key concepts of the textbooks and on-line quizzes giving formative feedback to the students. Such elements should thus be studied by the students before attending the synchronous sessions. Because of the preparatory work made by the students, the synchronous sessions can focus on much more active forms of learning under the teacher's supervision. The success of the pedagogical approach entirely depends on the contents of the synchronous sessions, which need to be carefully planned and designed so that they promote student learning. Although the hybrid learning environment gives rise to some additional challenges from a teacher's perspective, it also gives much more flexibility in attracting students from remote locations, without compromising the learning experience.
\end{abstract}

KEYWORDS: nuclear engineering education, active learning, flipped classroom, hybrid learning environment

\section{INTRODUCTION}

With overall declining student enrolments in nuclear engineering programs in Europe, being able to maintain highly specialized courses alive has become a challenge. As a possible remedy to such a situation, efforts have been pursued at Chalmers University of Technology, Gothenburg, Sweden to offer short courses in hybrid learning environments. Such environments make it possible to combine on-site and off-site attendees while preserving full interaction possibilities between both audiences and between each audience and the teacher. For that purpose, a special interactive teaching room was developed and allows both audiences to share audio, video and digital contents. Beyond the design of the room, special attention was put on implementing new pedagogical methods favoring student learning and interactions with the teacher.

This paper reports on two short courses arranged in this hybrid format: a course titled "Fundamentals of reactor kinetics and theory of small space-time dependent fluctuations in nuclear reactors" offered as part of the European Horizon 2020 CORTEX project (CORe monitoring Techniques and EXperimental validation and demonstration) [1], and a course titled "Deterministic modelling of nuclear systems" 
offered as part of the European Horizon 2020 ESFR-SMART project (European Sodium Fast Reactor Safety Measures Assessment and Research Tools) [2].

Both courses are given as "flipped" classes, i.e. the actual lectures are delivered asynchronously as short recorded lectures or webcasts. The students have to follow such webcasts prior to attending the synchronous sessions that are arranged either face-to-face for the on-site audience or remotely on the web for the off-site audience. Moreover, web-based quizzes associated to each webcast give the opportunity for the students to comprehend and further reflect on the topics presented during the webcasts. The main incentive in flipped classrooms is to get the students prepared to the synchronous sessions with the teachers, during which activities promoting higher-order thinking skills are arranged. Designing, implementing, and carrying out such sessions in hybrid environments is particularly strenuous. Nevertheless, student learning highly relies on the success of embedding active learning elements in the synchronous sessions. Beyond describing in more detail the set-up of both courses from a pedagogical perspective, the paper also focuses on the synchronous activities given in both courses. For the CORTEX course, those included short summarizing lectures or wrap-ups, discussions on the quizzes, and exercises requiring theoretical derivations led by the teacher. For the ESFR-SMART course, wrap-ups and discussions on the quizzes were also part of the synchronous sessions. In addition, the core of the active learning sessions was set up around the writing of various programming assignments in MATLAB Grader under the guidance of the teacher.

The paper is structured as follows. The two short courses are first described, focusing mostly on the pedagogical approach used and, when relevant, the required IT infrastructure. Thereafter, the synchronous sessions offered in both courses are analyzed both from the students' and the teacher's perspectives. Finally, the results of the course evaluations are analyzed. The paper thereafter concludes on the applicability of the proposed course set-up and makes some recommendations.

\section{DESCRIPTIONS OF THE COURSES}

The course titled "Fundamentals of reactor kinetics and theory of small space-time dependent fluctuations in nuclear reactors" was given on June 18-21, 2018 and had 16 on-site and 26 off-site registered participants, whereas the course titled "Deterministic modelling of nuclear systems" was given on September 9-13, 2019 and had 22 on-site and 39 off-site registered participants.

As customary when offering courses free of charge, not all registered participants did actually come on site or did participate to the courses remotely. In terms of on-site attendance, the first course attracted 14 students, while the second course attracted 11 students. All the on-site attendees successfully completed all the in-class assignments and obtained a course certificate. Since the remote students had the possibility to either work on the in-class assignments and correspondingly obtain a course certificate or only get hold of the course materials, no strict control of the participation of the remote attendees was carried out. Providing the actual number of remote attendees following all course moments is thus not possible. Nevertheless, a careful check of the completion of all in-class assignments by the remote attendees wishing to obtain a course certificate was carried out. The remote attendees who obtained a course certificate amounted to 10 students for the first course and to 16 students for the second course.

In both courses, the audience was mixed: MSc students with a solid background in nuclear engineering, $\mathrm{PhD}$ students and Post-Doc students in nuclear-related subjects, nuclear engineers and research scientists. Both courses were worth 1.5 ECTS (European Credit Transfer System). 


\subsection{Contents}

The first course covered the fundamentals of nuclear reactor kinetics, with emphasis on one- and twogroup diffusion theory and provided a solid and rigorous theoretical background in reactor dynamics. The course also presented a special case of reactor kinetics, i.e. small space-time stationary fluctuations in nuclear reactors, also referred to as neutron noise or power reactor noise. Attention was put in the course on the derivation of the governing equations and on how to solve such equations. The course was designed so that the course attendees, after completing the course, were able to:

- Know the governing equations describing reactor kinetics in diffusion theory.

- Know the governing equations describing power reactor noise in diffusion theory.

- Know how to solve such equations either analytically for homogeneous or piece-wise homogeneous systems, and numerically for heterogeneous systems.

The course was structured around two main chapters. The first chapter dealt with space-time dependent reactor kinetics in diffusion theory and covered the following topics:

- Static neutron transport (derivation of the static space-dependent neutron balance equations in diffusion theory, case of steady-state one-group diffusion theory, case of steady-state two-group diffusion theory).

- Dynamic neutron transport (derivation of the dynamic space-dependent neutron balance equations in diffusion theory, case of dynamic one-group diffusion theory, case of dynamic two-group diffusion theory).

- Resolution of the space- and time-dependence of the neutron flux in nuclear reactors (general discretization methods in space and time in diffusion theory, reduced Order Modelling - ROM in diffusion theory, flux factorization methods in diffusion theory).

The second chapter covered small space-time dependent fluctuations (power reactor noise) and the following specific topics:

- Theory of first-order neutron noise (general principles, derivation of the first-order neutron noise in one-group diffusion theory, derivation of the first-order neutron noise in two-group diffusion theory).

- Theory of first-order neutron noise in its factorized form (general principles, determination of the fluctuations of the amplitude factor, determination of the fluctuations of the shape function).

- General solution of the neutron noise in one-group diffusion theory.

- General solution of the neutron noise in two-group diffusion theory.

- Validity of the point-kinetic approximation (case of critical systems, case of subcritical systems with an external neutron source).

- Spatial discretization methods for resolving the neutron noise in nuclear reactors.

The second course covered the deterministic modelling of nuclear systems, with emphasis on neutron transport, fluid dynamics and heat transfer. This course aimed at presenting the main algorithms in the computer codes used by the industry and in academia for the macroscopic modelling of nuclear systems. The underlying methods used in such codes, together with their assumptions and limitations, were thoroughly presented, so that the codes could be used with confidence. The course was designed so that the course attendees, after completing the course, were able to:

- Know the governing equations describing neutron transport, flow transport, and heat transfer in nuclear reactors.

- Know the modelling strategies used for neutron transport, flow transport, heat transfer in nuclear reactors, and for their coupling.

- Understand the limitations of the different modelling strategies. 
The course was organized in six chapters. In the first introductory chapter, the governing equations for neutron transport, fluid transport, and heat transfer were derived, so that students not familiar with any of these fields could comprehend the course without difficulty. The peculiarities of nuclear reactor systems, i.e. their multi-physic and multi-scale aspects, were dealt with. An overview of the modelling strategies was thereafter given, with particular emphasis on deterministic methods, which represented the focus area of the course. In the second chapter, the computational methods for neutron transport at both the pin cell and fuel assembly levels were presented. The chapter was aimed at following the solution procedure in fuel pin/lattice codes as much as possible. This included resonance calculations of the cross-sections, the determination of the micro-region micro-fluxes, and of the macro-region macro-fluxes, and finally spectrum correction. The chapter ended with the preparation of the macroscopic cross-sections for subsequent core calculations, where the effect of burnup was also detailed. In the third chapter, the computational methods used for core calculations were presented. In the first part of this chapter, the treatment of the angular dependence of the neutron flux was described. In the second part, the treatment of the spatial dependence of the neutron flux was outlined. Thereafter, the solution procedure for estimating the core-wise position- (and possibly direction-) dependent multigroup neutron flux was described. Finally, the methodology used for determining the core-wise space- and time-dependent neutron flux in case of transient calculations was derived. The fourth chapter of the course focused on the computational methods used for one-/two-phase flow transport and heat transfer. From the local governing equations of fluid flow and heat transfer, macroscopic governing equations were derived, and the underlying assumptions clearly emphasized. The different flow models commonly used in nuclear engineering were introduced, models having various levels of sophistication: the two-fluid model, the mixture models with thermal equilibrium and specified drift, and the Homogeneous Equilibrium Model. The temporal and spatial discretization of the flow and heat transfer models were given special attention, with emphasis on their stability, consistence, and convergence. The fifth chapter tackled solving the coupling between neutronics and thermal-hydraulics at the core level. Various aspects of multi-physics coupling were highlighted: segregated versus monolithic approaches, coupling terms and non-linearities, information transfer, preparation of the macroscopic material data (cross-sections, diffusion coefficients, and discontinuity factors) as functions of the thermal-hydraulic variables, spatial coupling. The numerical techniques that could be used to solve multi-physics temporal coupling either in a segregated or in a monolithic manner were also discussed in detail. The last and sixth chapter summarized in, a nutshell, the macroscopic modelling techniques and presented a quick overview of the current efforts in high-fidelity reactor modelling.

\subsection{Pedagogical approach}

Both courses relied on an innovative pedagogical approach building upon the concept of active learning and a flipped classroom set-up.

According to Bloom's revised taxonomy for the cognitive domain, which is illustrated in Fig. 1, students go through various thinking skills while learning [3]. This process starts from low-order thinking skills, such as remembering and understanding the course concepts, to high-order thinking skills, such as applying, analyzing, evaluating the course concepts and creating. In a more traditional format, engineering students are exposed to new concepts for the first time in class. As a result, only low order thinking skills are triggered in the classroom. If the amount of new information provided to the students is too large, they will also have difficulty in processing the contents and will often become passive. This leads to the necessity for the students to go through the same contents as in the classroom but on their own and after the in-class sessions. Moreover, the students will have to deal with the course concepts at higher thinking orders mostly 
outside of the classroom and again on their own, unless dedicated in-class sessions are planned for such a purpose in the course curriculum.

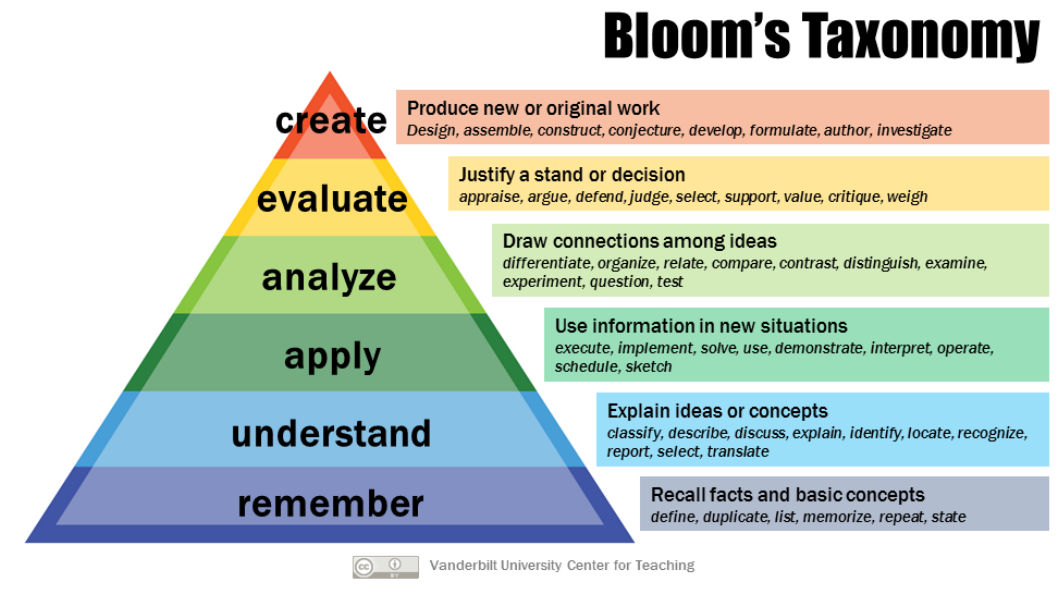

Fig. 1 - Illustration of Bloom's revised taxonomy for the cognitive domain, with higher-order thinking skills at the top of the diagram (from [4]).

In the flipped classroom model, students are asked to do some preparatory work before attending the inclass sessions. In this asynchronous learning phase, the students can choose when and at what pace to study the preparatory course material. In contrast to the traditional teaching format, low-order thinking skills are practiced during this asynchronous phase, before the students meet the teachers and other students for synchronous interactions. As a consequence, the time spent with the teachers can be used more effectively to engage students in high order thinking, clarify difficult concepts and provide individual support. Since the students attend the synchronous sessions much better prepared than in a traditional teaching set-up, flipped classrooms were demonstrated to lead to much better learning outcomes and to contribute to a deeper approach to learning compared to traditional teaching $[5,6]$.

The key aspect of flipped classrooms is freeing time in the classroom in order to organize engaging activities with the students under the teacher's supervision, thus favoring more active forms of learning. The active learning elements used in the two courses are thoroughly described in Section 3.

Another novelty of the course set-up was to offer the courses in a hybrid learning environment, i.e. the students could follow the courses either on-site or remotely on the web. From both a pedagogical and an implementation perspective, following the courses and training sessions remotely create some additional challenges. A dedicated interactive teaching room was then designed [7], so that the synchronous in-class active learning sessions could be broadcasted on the internet while preserving full interaction possibilities between the on-site audience, the teacher and the off-site audience. This room is furnished with movable chairs, tables and whiteboards enabling the use of a more student-centered pedagogical approach. In addition, the room is equipped with audio and video hardware and software ( 2 cameras, 4 ceiling microphones, 6 ceiling loudspeakers, and 1 portable microphone, all combined using a AV Bridge ${ }^{\mathrm{TM}}$ Matrix Pro). The core of the system is driven by a high-end tablet PC running web-based conferencing tools and connected to the Bridge. An additional screen aimed at handling the communication with the remote participants is connected to the tablet and a video projector is mimicking the screen of the tablet. This setup allows the tablet screen to be shared to the on-site attendees (via the projector) and to the off-site attendees (via the web-based conferencing tool). Because of the nature of the tablet, the teacher has the possibility to show slides, annotate them, and write on the screen, all of this being visible to the on-site and off-site students. Moreover, the audio/video equipment allows synchronous interactions between the on- 
site and off-site participants in form of digital content sharing, audio interactions, and video communication. A picture of the room set-up is shown in Fig. 2.

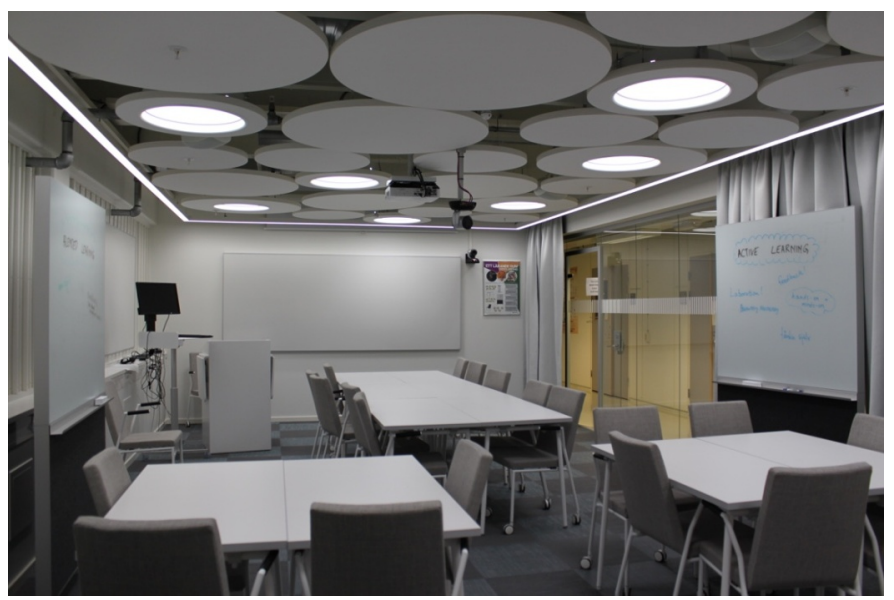

Fig. 2 - Picture of the interactive teaching room developed at Chalmers University of Technology (CAnna Wallin).

Such a teaching room allows offering the course to remote students in a pure web-based environment without any need to travel. The interactive teaching room allows guaranteeing the availability of active learning during the synchronous sessions for both the on-site and off-site attendees, who can furthermore interact between each other.

The entire pedagogical approach used in the two courses is summarized in Fig. 3. The students had first to study the textbooks specifically written for both courses. Short lectures or webcasts associated to each of the sections of each chapter were also recorded and made available to the students. Those lectures aimed at extracting the most important features presented in the respective textbooks in order to help the students construct a hierarchical and conceptual understanding of those features, with the details presented in the textbook and left for self-studying. The lectures were recordings of lecture slides accompanied by the oral narrative from the teacher and with on-screen annotations made by the teacher. On-line quizzes were also associated to each of the webcasts, in order to provide formative feedback to the students on their learning. The quizzes were designed in such a way that high order thinking skills in Bloom's revised taxonomy were solicited. The webcasts and on-line quizzes were made available online using a platform called Chalmers Play, itself based on the Kaltura platform. Those three first moments, i.e. study of the textbook, attendance of the webcasts, and training on the on-line quizzes, represent the preparatory work the students had to complete prior to attending the in-class sessions (either on-site or off-site). Only asynchronous interactions between the teacher and the students were possible during those three first moments. Synchronous interactions were only possible during the in-class sessions, using remote conferencing/webinar software (Adobe Connect for the first course and Zoom for the second course). The synchronous sessions and the corresponding active learning elements are further described in the next section.

\subsection{Active learning techniques in the synchronous sessions}

The synchronous sessions were made of two distinct elements: wrap-ups and activities involving the students.

The wrap-up were short lectures prepared in advance by the teacher and aimed at extracting from the various chapters the salient features of the concepts presented in the textbooks and in the webcasts. Those 
wrap-ups were specifically designed in order to help the students get a bird's eye view of the entire course and main concepts, thus further helping the students in establishing the inter-relations existing between the various topics covered. Furthermore, in case the students had not studied a given part of the textbook and the corresponding webcasts, the wrap-ups constituted a last opportunity for those students to catch up and comprehend most of the session following the wrap-up. After the actual lectures, discussions were initiated with the students either in an open "Question and Answer" session aimed at answering the questions raised by the students or in a more structured manner building upon the on-line quizzes the students were supposed to complete before the sessions.

Most of the time in the synchronous sessions were spent on more active forms of learning, during which the students were actively engaged in activities carefully prepared by the teacher. When using active learning techniques, students learn much more efficiently since they are in control of their learning in the classroom with the support from the teacher [8].

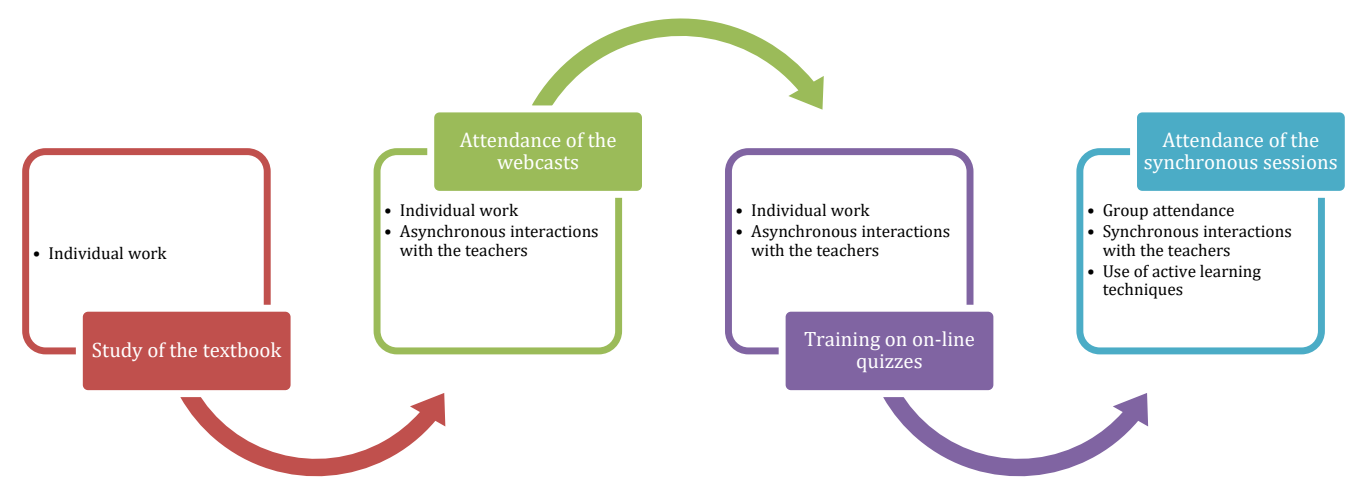

Fig. 3 - Summary of the pedagogical approach.

In the course "Fundamentals of reactor kinetics and theory of small space-time dependent fluctuations in nuclear reactors", the active learning technique that was used was group problem solving, a subcategory of collaborative learning. The students were put in groups of three or four, and they were assigned a task, question, or problem to solve together. Eight problems had been prepared on the first chapter of the course, and 12 problems had been prepared on the second chapter. Due to time constraints, the students only had time to go through half of the prepared exercises. The problems were of the "pen and paper" type, i.e. the students were asked to write down some theoretical derivations to find the answers to the problems. After being provided with instructions from the teacher, the students had to solve each of the problems. This was done in a collaborative manner between the students, as well as with the teacher, i.e. the teacher provided additional explanations and theoretical considerations when needed. The exercises were solved one after the other, i.e. the students were asked to complete each assignment at a pace dictated by the teacher. This allowed the teacher to also build upon each assignment, provide complementary information and most importantly relate the theoretical derivations to practical applications of reactor kinetics. Discussing the outcomes of each assignment was fundamental in capitalizing on the gained knowledge and soliciting high order thinking skills among students.

In the course "Deterministic modelling of nuclear systems", the active learning technique that was used was also based on group problem solving, although in the completely different set-up. Namely, seven coding assignments had been prepared by the teacher. Each coding assignment focused on some specific part of the textbook and the students were thus asked to implement the numerical techniques and algorithms to a practical case, which was a one-dimensional heterogeneous sodium-cooled reactor in steady-state conditions. After developing a cross-section model in eight energy groups, the students had to 
develop a diffusion-based neutron transport solver, a fluid dynamic solver for the liquid sodium, and a heat transfer model resolving the axial and radial distribution of the temperature in the fuel rods. Finally, the students had to solve the entire coupled problem in a tightly coupled manner using the Jacobian-Free Newton Krylov method, for which they had to first implement the algorithm. In total, the students had to go through seven coding assignments, the ultimate assignment allowing the students to compute the coupled neutronic/thermal-hydraulic solution to the considered sodium-cooled system. All coding assignments were carried out in MATLAB Grader, which is a web-based platform allowing the students to complement some MATLAB codes, test those, and submit their solutions when all tests were successfully performed. Because of its web-based nature, MATLAB Grader provided the exact same coding environment to both the on-side and off-site attendees. A 30 day-free trial version of the full desktop version of MATLAB was also provided to all students, in case they had not already access to MATLAB. The full desktop version of MATLAB gave much more flexibility as compared to MATLAB Grader in case the students wanted to further test their codes.

In both courses, the students were deeply engaged in solving the various assignments, discussing those with their peers and with the teacher. For the teacher, it is furthermore extremely rewarding to be able to help the students when they most need help. Solving the assignments also triggered numerous questions from the students. Although some of the questions were directly related to the assignments, some other ones were of much more general nature. Beyond the level of student engagement, the level of the questions also demonstrated that the students learned much better in this teaching format with a deeper learning of the subject and utilized higher order thinking skills in Bloom's revised taxonomy.

\section{ANALYSIS OF THE COURSE EVALUATIONS}

For both courses, an identical course evaluation questionnaire was used at the end of each course. For the first course, 23 persons responded to the course evaluation, out of which $52.2 \%$ were on-site participants. For the second course, 25 persons responded to the course evaluation, out of which $40 \%$ were on-site participants.

Fig. 4 represents the respondents' overall impression on the courses, where one notices that all respondents considered the course to be either good or, to an overwhelming fraction, very good. No respondent was dissatisfied with the courses. The respondents were also asked to determine whether they learned better in the flipped classroom format or in a more traditional teaching format. As Fig. 5 demonstrates, a large majority of the respondents $(73.9 \%$ for the first course and $68 \%$ for the second course) believed that they learned better or much better in the flipped classroom format than in the traditional format. Moreover, as Fig. 6 reveals, the quality of the pedagogical approach followed in the courses was considered to be either good or very good (to an overwhelming fraction for the first course).

Finally, the students were asked to determine whether the on-line quizzes contributed positively or negatively to their learning (see Fig. 7) and whether they found the synchronous sessions engaging (see Fig. 8). The students overwhelmingly considered that the on-line quizzes favored their learning and that the synchronous sessions were engaging (with a vast majority in the second courses considering that the sessions were very engaging). A closer examination of the additional comments provided by the students demonstrated that, for the first course, dialogue with the teacher was somehow limited. This was explained by the fact that handling both the questions from the remote and on-site attendees represents a very challenging situation for the teacher, especially when the questions from the remote attendees are numerous and come from several sources (audio communication, chat room, Q\&A). To circumvent this difficulty, help from a teaching assistant was obtained in the second course. The main responsibility of the teaching assistant was to handle the communication with the remote students and help those students when needed. Having an additional resource in the second course might explain the increase in student engagement. 


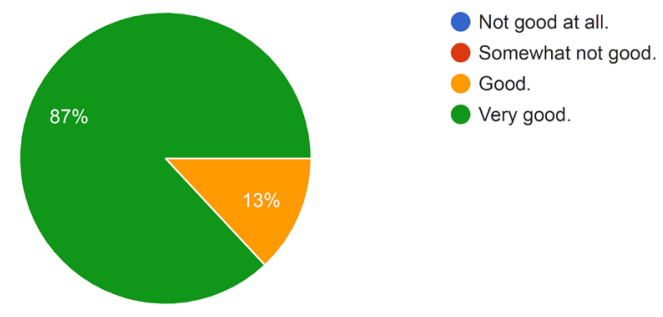

Course "Fundamentals of reactor kinetics and theory of small space-time dependent fluctuations in nuclear reactors"

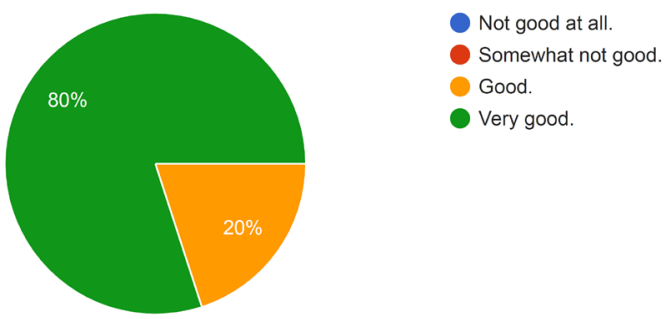

Course "Deterministic modelling of nuclear systems"

Fig. 4-Overall impression on the courses.

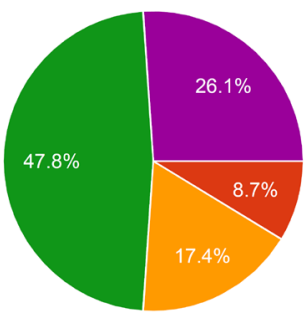

Course "Fundamentals of reactor kinetics and theory of small space-time dependent fluctuations in nuclear reactors"
Much better in the traditional set-up

Somewhat better in the traditional set-up

Equally well in the traditional and flipped set-ups

Somewhat better in the

flipped set-up

Much better in the flipped set-

up

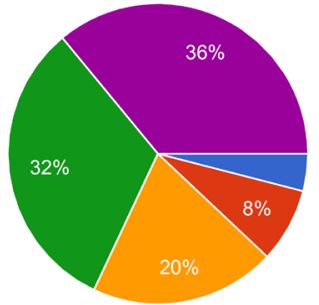

Much better in the traditional set-up

Somewhat better in the traditional set-up

Equally well in the traditional and flipped set-ups

Somewhat better in the flipped set-up

Much better in the flipped setup

Course "Deterministic modelling of nuclear systems"

Fig. 5 - Teaching format best adapted to learning the concepts of the courses.

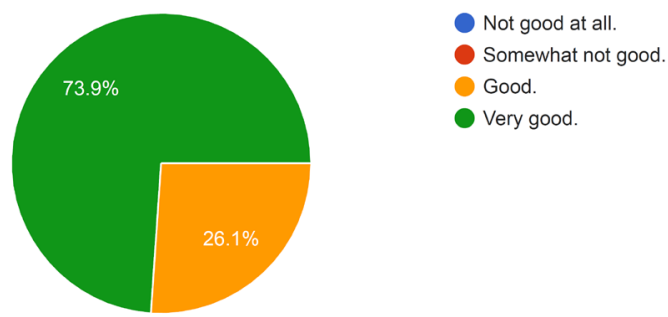

Course "Fundamentals of reactor kinetics and theory of small space-time dependent fluctuations in nuclear reactors"

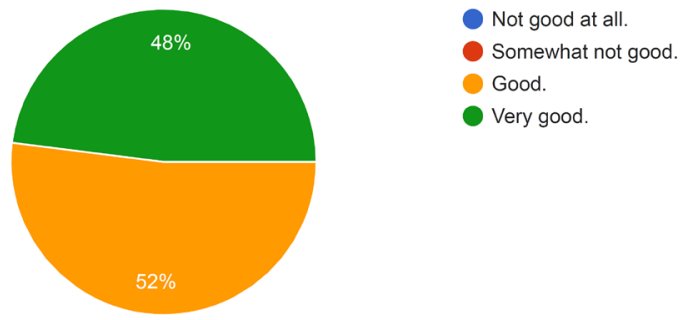

Course "Deterministic modelling of nuclear systems"

Fig. 6-Quality of the pedagogical approach followed in the courses. 


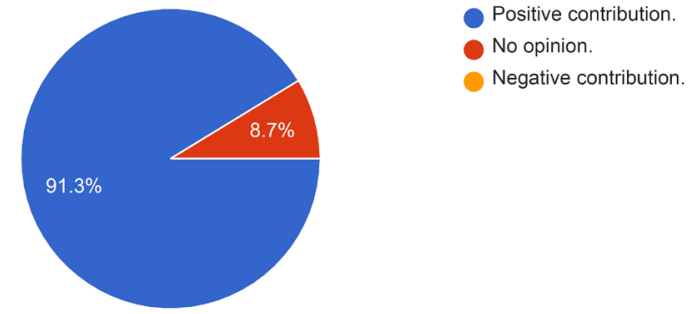

Course "Fundamentals of reactor kinetics and theory of small space-time dependent fluctuations in nuclear reactors"

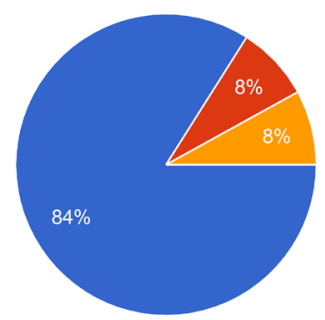

Positive contribution

No opinion.

Negative contribution.

Fig. 7 - Contribution from the on-line quizzes to learning.

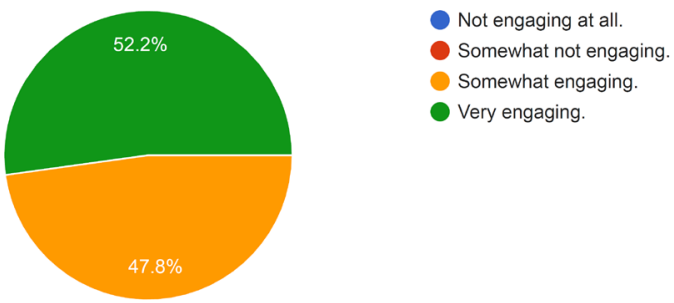

Course "Fundamentals of reactor kinetics and theory of small space-time dependent fluctuations in nuclear reactors"

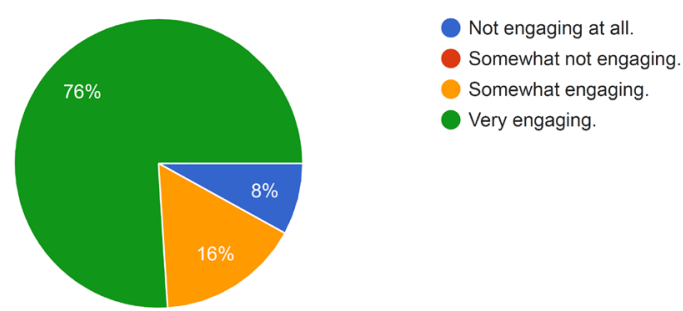

Course "Deterministic modelling of nuclear systems"

Fig. 8 - Engagement in the synchronous sessions.

\section{CONCLUSIONS AND RECOMMENDATIONS}

As demonstrated in this paper, student-centered teaching approaches favor a deeper understanding of the presented topics, thanks to the flipped nature of the set-up and the active learning techniques used in the synchronous sessions. Compared to a traditional teaching format, the proposed set-up leads to much more interactions between all parties involved, even if the courses are partially offered on-line.

The flexibility with the hybrid format and with self-paced learning thanks to the flipped classroom make the course offering particularly attractive to students who do not have the possibility to travel and to staff members in the nuclear sector who cannot always come on-site to follow a course. In addition, the 24/7 availability of the recorded lectures and electronic resources is an aspect making this teaching format particularly well suited for continuous education of staff members and life-long learning.

It should nevertheless be mentioned that the development of such a hybrid course with such an innovative pedagogical concept requires careful preparation and planning, and foremost, dedication from the teacher undertaking such a radical transformation. In addition to the necessary time and required efforts, the technical and administrative frameworks in place at the respective university might not be adapted to the teaching format. Moreover, many IT resources are required, such as a streaming platform for the webcasts, a platform for the quizzes, a platform for the remote synchronous sessions, and a platform for e.g. the coding 
assignments. Learning all those resources and obtaining the necessary help from the competent/responsible persons might also represent some additional complications and create some additional delays when setting up all those electronic resources. Furthermore, because of the asynchronous nature of most of the resources being made available to the students, those resources need to be ready well ahead of the synchronous sessions. Thorough testing of those resources is also necessary before they are made available to the students.

Despite the challenges of using a hybrid learning environment, this innovative concept might represent a viable alternative to either fully on-site or fully web-based courses. This is particularly interesting considering the decreasing number of students enrolled in nuclear engineering programs at European universities. Thanks to the web-based character of the course, it is possible to attract a sufficient number of students, by combining the on-site and off-site attendees.

\section{ACKNOWLEDGMENTS}

The research conducted was made possible through funding from the Euratom research and training programme 2014-2018 under grant agreement No 754316 (CORTEX project) and under grant agreement No 754501 (ESFR-SMART project).

\section{REFERENCES}

1. C. Demazière et al., "Overview of the CORTEX project," Proceedings of the International Conference on the Physics of Reactors - Reactor Physics paving the way towards more efficient systems (PHYSOR2018), Cancun, Mexico, April 22-26, 2018 (2018).

2. K. Mikityuk et al., "ESFR-SMART: new Horizon-2020 project om SFR safety," Proceedings of the International Conference on Fast Reactors and Related Fuel Cycles: Next Generation Nuclear Systems for Sustainable, Development (FR17), Yekaterinburg, Russia, June 26-29, 2017 (2018).

3. L.W. Anderson et al., A taxonomy for learning, teaching, and assessing: A revision of Bloom's taxonomy of educational objectives, Pearson, Allyn \& Bacon (2000).

4. The Vanderbilt University Center for Teaching, "Bloom's taxonomy," available online. URL https://cft.vanderbilt.edu/guides-sub-pages/blooms-taxonomy/ (2017).

5. J. O'Flaherty and C. Phillips, "The use of flipped classrooms in higher education: A scoping review," The Internet and Higher Education, 25, pp. 85-95 (2015).

6. J.L. Bishop and M.A. Verleger, "The flipped classroom: A survey of the research," ASEE National Conference Proceedings, Atlanta, GA, USA, 30 (9) (2013).

7. C. Demazière et al., "Setting up a room to cater to online learners' needs," Proceedings of the Conference on Nuclear Training and Education (CONTE 2017), Jacksonville, FL, USA, February 5-8, 2017 (2017).

8. S. Freeman et al., "Active learning increases student performance in science, engineering, and mathematics," Proceedings of the National Academy of Sciences, 111 (23), pp. 8410-8415 (2014). 